\title{
Bacterial translocation: impact of probiotics
}

\author{
Bengt Jeppsson', Peter Mangell', Diya Adawi' and Göran Molin ${ }^{2}$ \\ 'Department of Surgery, Malmö University Hospital, and ²Department of Food Technology, Lund University, Lund, \\ Sweden
}

Abstract

There is a considerable amount of data in humans showing that patients who cannot take in nutrients enterally have more organ failure in the intensive care unit, a less favourable prognosis, and a higher frequency of septicaemia, in particular involving bacterial species from the intestinal tract. However, there is little evidence that this is connected with translocation of bacterial species in humans. Animal data more uniformly imply the existence of such a connection. The main focus of this review is to describe different ways to alter the luminal milieu to decrease bacterial translocation. It is possible to reduce absorption of endotoxin by administration of bile salts in obstructive jaundice. Increasing the oral intake of glutamine will reduce bacterial translocation in rats with radiation-induced gut injury. The bacterial microflora plays a very important role in maintaining the normal intestinal ecological environment and supplying preferred fuels to the intestinal wall, consequently supporting the intestinal barrier. Disruption of the balance of intestinal bacterial microflora may increase the incidence of bacterial translocation by modifying intestinal barrier function. Bacterial species such as enteric Gram-negatives and Gram-positive cocci are more prone to translocation, whereas lactobacilli seem to have a protective effect. Administration of live lactobacilli either orally or by enema will reduce translocation. The mechanisms underlying the decreased translocation are not obvious. One effect may be mediated via an action on the intestinal wall and its permeability. Recently, the results of three randomized studies on the use of L. plantarum in patients with pancreatitis, undergoing liver transplantation or upper gastrointestinal surgery have been published, which all indicate a potential role for lactobacilli in translocation. In conclusion, by altering the luminal content of bacteria it seems possible to reduce the incidence of secondary infections. The influence of the luminal milieu on bacterial translocation is not fully understood. There is convincing evidence from experimental studies, but only circumstantial evidence from clinical studies.

Keywords: Bacterial translocation; barrier function; lactobacilli; probiotics.

Received: 19 Jan. 2004; Accepted: 21 Jan. 2004

It is widely accepted that, whenever possible, nutritional support should be given enterally, reserving parenteral nutrition for patients with intestinal failure (1), since the absence of luminal nutrition has deleterious effects on intestinal morphology, barrier function and immune stimulation. This is especially marked in patients in the intensive care unit (ICU) where there is some evidence for an increased presence of microbial DNA of enteric origin in the blood (2). This is ascribed to bacterial translocation, which is defined as "passage of both viable and non-viable microbes and microbial products such as endotoxin across the anatomically intact intestinal barrier".

There is a considerable amount of data in humans showing that patients who cannot take in nutrients by the enteral route have more organ failure in the ICU, a less favourable prognosis, and a higher frequency of septicaemia, in particular involving bacterial species from the intestinal tract. However, there is little evidence that this is connected with translocation of bacterial species in humans (3).

Animal data more uniformly imply the existence of such a connection. One must bear in mind, however, that there may be species differences and regional differences in the gut regarding permeability changes and translocation (4), making it difficult to extrapolate findings from animals to humans. In addition, bacterial translocation occurs naturally to some extent and this makes it difficult to judge when it has a negative impact on the host. 


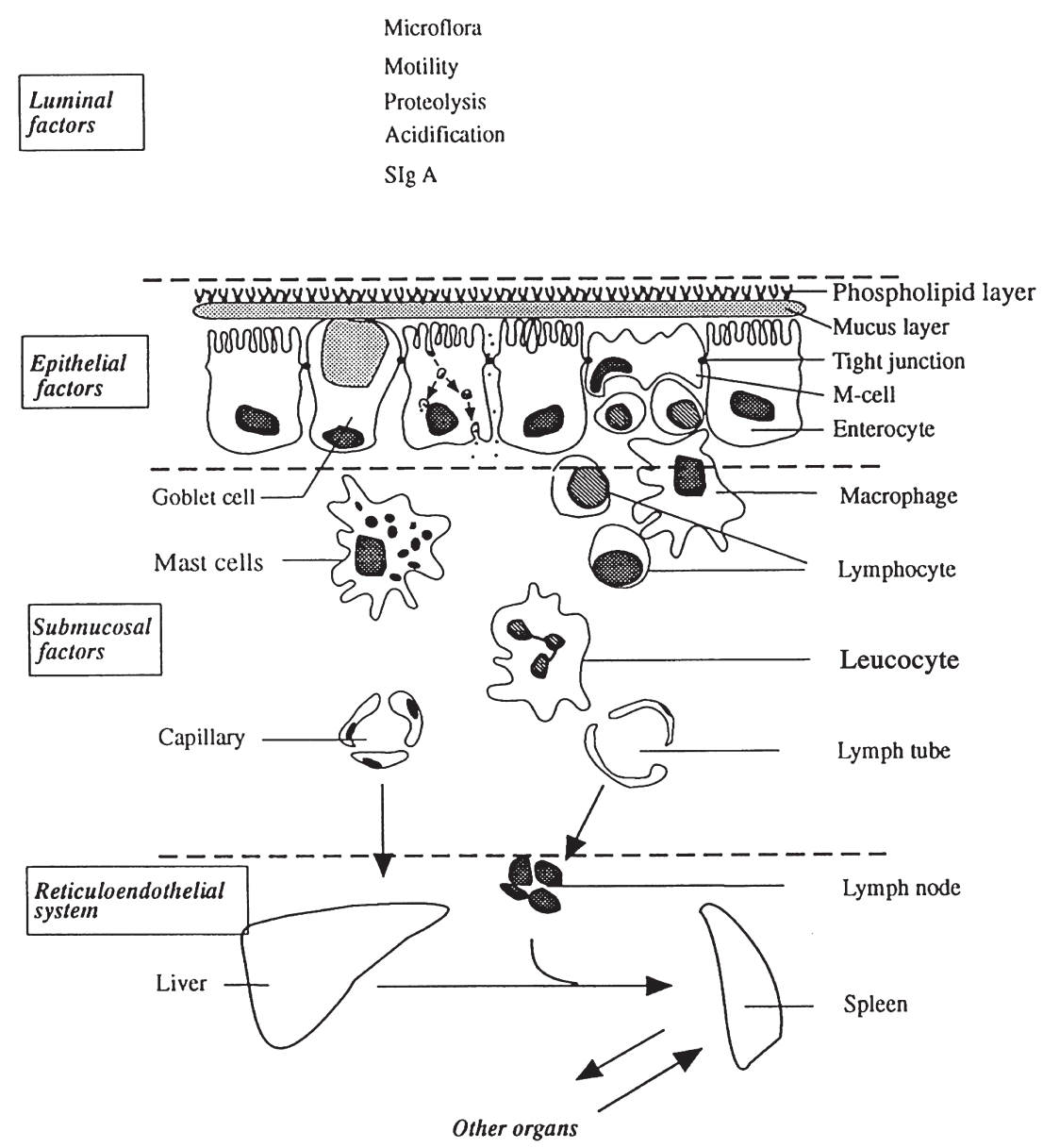

Fig. 1. Schematic presentation of the gastrointestinal defence barrier and effector factors. Soluble Ig A (SIg A).

The barrier function of the gastrointestinal tract is maintained by the interaction of several factors from luminal content to different components in the intestinal wall to different functions beyond the wall and the reticuloendothelial system (Fig. 1, Table 1). There is a considerable input of the immune system. At present, which components are disturbed in different pathogenic situations is not known, and little is known about how to correct them. One part can rather easily be affected and that is the luminal content.

The main focus of this review is therefore to describe different ways to alter the luminal milieu to decrease bacterial translocation. Most emphasis in this respect has been placed on the bacterial luminal content, but there is some evidence that a normal content of bile is important and that some nutrients in the gut are important, e.g. glutamine and arginine (5).

The human gastrointestinal tract contains bacterial cells at an equivalence of $1 \mathrm{~kg}$ per person. There is a marked variation in the number and pattern of bacterial flora at different levels of the tract. The number of ingested bacteria is reduced dramatically by gastric acid. A low concentration of organisms is maintained in the small bowel by intestinal motility, which ensures that non-adherent organisms are constantly cleared from the small bowel. Despite this clearing, the concentration of bacteria increases from the jejunum through the ileum, with a remarkable elevation in the distal small bowel and large bowel (6).

The microflora plays a very important role in maintaining the normal intestinal ecological environment and supplying preferred fuels to the intestinal wall, consequently supporting the intestinal barrier (7). Changes in diet, stress, the use of antibiotics and excessive hygiene all bring changes in the microbiological ecosystem. Studies have shown that disruption of intestinal microflora balance may increase the incidence of bacterial translocation by modifying intestinal barrier function (7). Interactions between the normal microflora and the intestinal mucosa combine to limit the 
Table I. Overview of the intestinal defence barrier

\begin{tabular}{lll}
\hline Factors & Non-specific defence & Immunological defence \\
\hline Luminal & Microflora & Slg A \\
& Motility & \\
& Proteolysis & \\
Ecidification & M-cells \\
& Hydrophobicity & Lymphocytes \\
& Mucous layer & \\
& Epithelial cell turnover & \\
& Microvilli and apical membrane & \\
& Tight junctions & Lymphocytes: \\
& Blood flow & B-cells \\
& Capillary endothelium & T-cells \\
& & Mast cells \\
& & Macrophages: \\
& & Phagocytes \\
& & Dendritic cells \\
& & Leucocytes: \\
& & Mononuclear cells \\
& Neutrophils \\
& & Eosinophils \\
& & Spleen \\
& & \\
& &
\end{tabular}

presence and binding of pathogenic bacteria to the intestinal epithelium.

Alterations in the luminal bacterial content alone will affect translocation. Any bacteria in the intestine showing high counts will give rise to more translocation (8). Bacterial species such as enteric Gram-negatives and Gram-positive cocci are more prone to translocation, whereas lactobacilli seem to have a protective effect (9). In general, probiotics containing fewer pathogenic strains seem to not translocate in themselves, at least not to any significant degree (10). In the rat the presence of bulk in the intestine also seems to prevent translocation (11).

The effect of dietary fibre and lactobacilli on bacterial translocation has been investigated in several studies. In models of cytotoxic drug-induced gut injury and liver injury, pectin and oat fibre were shown to reduce bacterial translocation $(11,12)$. Administration of live lactobacilli either orally or by enema will further reduce the translocation (12). The effect is different for different lactobacilli and the strongest effect was found with Lactobacillus plantarum and L. rhamnosus (13). Similarly, in a model of pancreatitis bacterial translocation was reduced by pretreatment with $L$. plantarum (14). A reduced rate of infected pancreatic necrosis could be observed as an effect of the reduced translocation.
The mechanisms underlying the decreased translocation are not obvious. One effect may be mediated via an action on the intestinal wall and its permeability. A study using the Ussing chamber technique found that lactobacilli will significantly reduce an Escherichia coli-induced increase in permeability (15). The dose and timing seem important.

Administration of L. plantarum in a study of liver injury verified the reduction in bacterial translocation. This could not be detected on mucosal samples after administration, but there was a significant increase in Lactobacillus species in general and thus one reason for the decreased translocation could be that the treatment with $L$. plantarum stimulated the resident Lactobacillus flora on behalf of other groups of bacteria (16).

There is no direct evidence that administration of probiotics will have an impact on bacterial translocation. One study has tried to address the problem (17). Patients undergoing elective major abdominal surgery were randomized to either a treatment or a control group. The treatment group received an oral preparation containing L. plantarum. There was no significant difference in terms of bacterial translocation. The dose of lactobacilli was low, much lower than in other experimental or clinical studies. Furthermore, the incidence of bacterial translocation in elective surgery is very low and this will require large studies to verify.

Recently, the results of three randomized studies on the use of $L$. plantarum in patients with pancreatitis, undergoing liver transplantation or upper gastrointestinal surgery, have been published, which all indicate a potential role of lactobacilli on translocation.

In a prospective double-blind randomized study the administration of L. plantarum 299 and oat fibre was compared with the administration of heat-killed L. plantarum 299 and oat fibre in patients with severe pancreatitis to investigate whether secondary infection of pancreatic necrosis by Gram-negative bacteria could be avoided. It has been shown that infection of pancreatic tissue is usually preceded by colonization of the large intestine by Gram-negative bacteria (18).

Administration of probiotics was started within $48 \mathrm{~h}$ of admission to the hospital. Out of 45 patients entering the study 22 had been given probiotics and 23 a placebo. Infected pancreatic necrosis and abscesses occurred in out of the 22 patients $(4.5 \%)$ 
in the lactobacilli group and in seven of the 23 patients $(30 \%)$ in the placebo group (18). Both groups received antibiotics. Although there was no complete microbiological work-up in the studied patients, the results indicate a new approach to the treatment of secondary infections of enteric origin in patients with severe inflammation. The results are similar to the experimental study mentioned above.

Another study in human liver transplant patients compared selected bowel decontamination in combination with standard enteral formula, administration of live L. plantarum 299 and oat fibre, and heat-killed L. plantarum 299 and oat fibre (19). The sepsis rate was $48 \%$ in the selected bowel decontamination group, $34 \%$ in the group treated with heat-killed lactobacilli and $13 \%$ in the group receiving live lactobacilli. The mean duration of antibiotic therapy, total hospital stay and stay in ICU were shorter in the group administered live lactobacilli.

In a third study from the same group, the effect of L. plantarum 299 was compared with heat-killed lactobacilli in the same dose in patients undergoing elective abdominal operations such as hepatic resection, pancreatic resection, gastric resection, colonic resection and intestinal bypass (20). Control patients received parenteral nutrition according to routine procedures in the department. The groups treated with either live or heat-killed lactobacilli suffered fewer infections: three out of 30 patients in each group $(10 \%)$ compared with nine out of 30 $(30 \%)$ in the parenteral group. An even larger difference was observed when the subgroups of gastric and pancreatic surgical patients were analysed.

In all these instances there is a risk of secondary infections of bacteria of enteric origin, as a result of the surgical trauma or ongoing infection. By altering the luminal content of bacteria it seems possible to reduce the incidence of secondary infections. Although these results need to be confirmed in larger prospective studies they indicate a new concept of altering the luminal bacterial milieu, resulting in reduced secondary infections.

The influence of the luminal milieu on bacterial translocation is still not fully understood. There is convincing evidence from experimental studies, but only circumstantial evidence can be found from clinical studies.

\section{References}

1. Jenkins AP, Thompson H. Enteral nutrition and small intestine. Gut 1994; 35: 1765-9.

2. Kane TD, Alexander JW, Johannigman JA. The detection of microbial DNA in the blood: a sensitive method for diagnosing bacteremia and/or bacterial translocation in surgical patients. Ann Surg 1998; 227: 1-9.

3. Lemaire LCJM, van Lanschot JJP, Stoutenbeele CP, Van Deventer SJH, Wells CL, Gouma DJ. Bacterial translocation in multiple organ failure: cause or epiphenomenon still unproven. Br J Surg 1997; 84: 1340-50.

4. Nejdfors P, Ekelund M, Jeppsson B, Weström B. Mucosal in vitro permeability in the intestinal tract in pig, rat, and man: species- and region-related differences. Scand J Gastroenterol 2000; 35: 501-7.

5. Ersin S, Tuneyurell P, Esassolak M, Alkanat M, Buke C, Yilmaz $M$, et al. The prophylactic and therapeutic effects of glutamine- and arginine-enriched diets on radiation-induced enteritis in rat. J Surg Res 2000; 89: 121-5.

6. Char S, Farthiny MJG. Bacteria and gut immunity. Curr Opin Gastroenterol 1994; 10: 659-63.

7. Nettelbladt CG, Katonli M, Bark T, Svenberg T, Mölllby R, Ljungqvist D. Bulking fibre prevents translocation to mesenteric lymph nodes of an efficiently translocating Escherichia coli strain in rats. Clin Nutr 1998; 17: 185-90.

8. Yu P, Martin CM. Increased gut permeability and bacterial translocation in Pseudomonas pneumoniaeinduced sepses. Crit Care Med 2000; 28: 2573-7.

9. Wenzl HH, Schimpl G, Feierl G, Stein-Wender G. Time course of spontaneous bacterial translocation from gastrointestinal tract and its relationship to intestinal microflora in conventionally reared infant rats. Dig Dis Sci 2000; 46: 1120-6.

10. Zhon JS, Shu Q, Rutherford KJ, Prasad J, Gopal PJ, Gill HS. Acute oral toxicity and bacterial translocation studies on potentially probiotic strains of lactic acid bacteria. Food Chem Toxicol 2000; 38: 153-61.

11. Mao Y, Nobaek S, Behzad K, Adawi D, Stenram U, Molin G, et al. The effects of Lactobacillus strains and oat fiber on methotrexate-induced entercolitis in rats. Gastroenterology 1996; 111: 334-44.

12. Adawi D, Kasravi FB, Molin G, Jeppsson B. Effect of Lactobacillus supplementation with and without arginine on liver damage and bacterial translocation in an acute liver injury model in the rat. Hepatology 1997; 25: $642-7$.

13. Mao Y, Nobaek S, Adawi D, Molin G, Jeppsson B. Comparison of the effects of different strains of Lactobacillus in reducing bacterial translocation on methotrexate-induced enterocolitis in rats. Dig Surg 1997; 14: 284-91.

14. Mangiante G, Colucci G, Canepari P, Bassi C, Nicoli N, Casaril A, et al. Lactobacillus plantarum reduces infection of pancreatic necrosis in experimental acute pancreatitis. Dig Surg 2001; 18: 47-50.

15. Mangell P, Nejdfors P, Wang M, Ahrné S, Weström B, Thorlacius $\mathrm{H}$, et al. Lactobacillus plantarum 299v 
inhibits Escherichia coli-induced intestinal permeability. Dig Dis Sci 2002; 47: 511-6.

16. Wang M, Adawi D, Molin G, Pettersson B, Jeppsson B, Ahrné S. Identification of the translocating bacteria in rats with acute liver injury and their relation to the bacterial flora of the intestinal mucosa. APMIS 2001; 109: 551-8.

17. McNaught CE, Woodcock NP, MacFie J, Mitchell CJ. A prospective randomized study of the probiotic Lactobacillus plantarum $299 \mathrm{v}$ on indices of gut barrier function in elective surgical patients. Gut 2002; 51: 827-31.

18. Oláh A, Belágyi T, Issekutz Á, Garnal ME, Bengmark S. Randomized clinical trial of specific lactobacillus and fibre supplement to early enteral nutrition in patients with acute pancreatitis. Br J Surg 2002; 89: 1103-7.
19. Rayes N, Seehofer D, Hansen S, Boucsein K, Müller AR, Serke S, et al. Early enteral supply of Lactobacillus and fibre vs selective bowel decontamination (SBD) - a controlled trial in liver transplants recipients. Transplantation 2002; 74: 123-7.

20. Rayes N, Hansen S, Seehofeer D, Müller A R, Serke S, Bengmark S, et al. Early enteral supply of fibre and lactobacilli vs conventional nutrition: a controlled trial in patients with major abdominal surgery. Nutrition 2002; 18: 609-15.

Bengt Jeppsson, MD, PhD, FRCS

Department of Surgery

Malmö University Hospital

SE-205 02 Malmö

Sweden

E-mail: bengt.jeppsson@kir.mas.lu.se 